\title{
A simple modification of TG-43 based brachytherapy dosimetry with improved fitting functions: Application to the selectSeed source
}

\author{
Xavier J. Juan-Senabre ${ }^{a}$, Ignacio Porras ${ }^{\mathrm{b}, *}$, Antonio M. Lallena ${ }^{\mathrm{b}}$
}

\author{
a Servicio de Radiofísica y Protección Radiológica, Consorcio Hospitalario Provincial de Castellón, \\ Av. Dr Clarà, 19. E-12002 Castellón de la Plana, Spain \\ b Departamento de Física Atómica, Molecular y Nuclear, Facultad de Ciencias, \\ Universidad de Granada, E-18071 Granada, Spain
}

Received 12 January 2012; received in revised form 10 May 2012; accepted 18 May 2012 Available online 12 June 2012

\section{KEYWORDS \\ TG-43; \\ ${ }^{125}$ I brachytherapy \\ sources; \\ Nucletron \\ selectSeed; \\ Monte Carlo; \\ PENELOPE}

\begin{abstract}
A variation of TG-43 protocol for seeds with cylindrical symmetry aiming at a better description of the radial and anisotropy functions is proposed. The TG-43 two dimensional formalism is modified by introducing a new anisotropy function. Also new fitting functions that permit a more robust description of the radial and anisotropy functions than usual polynomials are studied. The relationship between the new anisotropy function and the anisotropy factor included in the one-dimensional TG-43 formalism is analyzed. The new formalism is tested for the ${ }^{125} \mathrm{I}$ Nucletron selectSeed brachytherapy source, using Monte Carlo simulations performed with PENELOPE. The goodness of the new parameterizations is discussed. The results obtained indicate that precise fits can be achieved, with a better description than that provided by previous parameterizations. Special care has been taken in the description and fitting of the anisotropy factor near the source. The modified formalism shows advantages with respect to the usual one in the description of the anisotropy functions. The new parameterizations obtained can be easily implemented in the clinical planning calculation systems, provided that the ratio between geometry factors is also modified according to the new dose rate expression. (c) 2012 Associazione Italiana di Fisica Medica. Published by Elsevier Ltd. All rights reserved.
\end{abstract}

\footnotetext{
* Corresponding author.

E-mail address: porras@ugr.es (I. Porras).
} 


\section{Introduction}

The AAPM Task Group no. 43 (TG-43) [1,2] proposed a protocol to calculate the dose delivered by a single brachytherapy seed. Specifically, two different approaches corresponding to two-dimensional (2D) cylindrically symmetric line sources and one-dimensional (1D) point sources were given.

Both approaches include a series of parameters and functions that are used by the clinical treatment planning systems to compute the source dose distributions. In fact, these systems perform the calculations using polynomials describing the functions of interest or data tables obtained from Monte Carlo (MC) calculations which are conveniently interpolated. Data for various sources are available in [2].

In this work we propose a variant of the 2D protocol, in which all the angular dependence is included in a new anisotropy function, and new fitting functions for the dosimetry factors appearing in the dose rate equations.

The new anisotropy function permits a simpler description of the angular dependence and allows establishing a more straightforward link between 2D and 1D formalisms.

As polynomials, commonly employed to describe the dosimetric functions, can be used safely only in the range where they were fitted, we propose new fitting functions that show a good behavior outside the fitting region, describe in a better way the functions of interest and have a number of free parameters similar to those previously used.

Special attention has been paid to the evaluation of the anisotropy factor, required for the 1D formalism. TG-43 [1,2] suggests calculating this factor by means of a numerical integration involving the 2-D anisotropy function and the geometry factor. In this work an alternative way of evaluating the anisotropy factor is presented. This permits to calculate this factor at short distances from the source accurately.

To illustrate the possibilities of the modifications proposed, the various dosimetric parameters and functions have been calculated and analyzed for the ${ }^{125}$ I Nucletron selectSeed brachytherapy source, using Monte Carlo simulations performed with PENELOPE [3]. The values obtained for the different TG-43 parameters and functions have been reported and compared to the available information [4-9].

\section{Materials and methods}

\section{Modified formalism}

TG-43 formalism [1,2] proposes to calculate the dose rate at a point of coordinates $(r, \theta)$ as:

$\dot{D}^{(2 \mathrm{D})}(r, \theta)=S_{K} \Lambda \frac{G_{\mathrm{L}}(r, \theta)}{G_{\mathrm{L}}\left(r_{0}, \theta_{0}\right)} g_{\mathrm{L}}(r) F(r, \theta)$,

for the 2D approach, and

$\dot{D}_{\mathrm{L}}^{(1 \mathrm{D})}(r)=S_{K} \Lambda \frac{G_{\mathrm{L}}\left(r, \theta_{0}\right)}{G_{\mathrm{L}}\left(r_{0}, \theta_{0}\right)} g_{\mathrm{L}}(r) \phi_{\mathrm{an}}(r)$

or

$\dot{D}_{\mathrm{P}}^{(1 \mathrm{D})}(r)=S_{K} \Lambda \frac{G_{\mathrm{P}}(r)}{G_{\mathrm{P}}\left(r_{0}\right)} g_{\mathrm{P}}(r) \phi_{\mathrm{an}}(r)$.

for the 1D one. In these expressions, $\theta_{0}=90^{\circ}$ and $r_{0}$ is a reference distance (usually $1 \mathrm{~cm}$ ). $S_{K}$ denotes the air kerma strength, $\Lambda$ the dose rate constant, $G_{L}(r, \theta)$ and $G_{P}(r, \theta)$ are the linear and point geometry factors, respectively, $g_{\mathrm{P}}(r)$ and $g_{\mathrm{L}}(r)$ are radial dose functions, $F(r, \theta)$ is the anisotropy function and $\phi_{\text {an }}(r)$ is the so-called anisotropy factor. Details about the definitions of these functions and factors can be found in $[1,2]$.

For the practical application of the formalism, TG-43 recommends $[1,2]$ to use a fitting polynomial (usually of fifth degree) describing $g_{\mathrm{L}}(r)$ and $g_{\mathrm{P}}(r)$ and data tables to be interpolated for $F(r, \theta)$. Some authors, see e.g [10]., suggested to consider more elaborated fitting functions, providing a better description of the quantities of interest. These authors proposed a fitting function that includes the exponential attenuation of the dose and the scatter contributions to it, for the radial dose function, while for the anisotropy function, a fitting function depending of an angular coordinate was assumed. From the practical point of view, this approach appeared to be rather useful for treatment planning systems.

For the anisotropy factor, required in the 1D formalism, TG-43 recommends $[1,2]$ to calculate it by performing the numerical integration of $G_{\mathrm{L}}(r, \theta) F(r, \theta)$. The main problem is the scarce number of data points of this product available for a given $r$ value, what makes the integration quite uncertain, as Furhang and Anderson pointed out [10]. In addition, and as indicated in TG-43, there are not much data available, experimental or $M C$, for $r<0.25 \mathrm{~cm}$ and for that short radii, the points for polar angles close to $0^{\circ}$ and $180^{\circ}$ are inside the capsule of the source, what avoids a right definition of the integration angular interval. This makes that $\phi_{\mathrm{an}}(r)$ is not very well known as a function of the distance from the source for most of the commercial seeds, and it is affected by large uncertainties. This is particularly important nearby the source, where large uncertainties may occur when the values for larger distances are extrapolated.

The modified approach that we propose for the 2D formalism is as follows:

$\dot{D}^{(2 \mathrm{D})}(r, \theta)=S_{K} \Lambda \frac{G_{\mathrm{L}}\left(r, \theta_{0}\right)}{G_{\mathrm{L}}\left(r_{0}, \theta_{0}\right)} g_{\mathrm{L}}(r) H_{\mathrm{L}}(r, \theta)$.

The main point is that all the angular dependence of the dose rate is included in the new anisotropy function

$H_{\mathrm{L}}(r, \theta)=\frac{G_{\mathrm{L}}(r, \theta)}{G_{\mathrm{L}}\left(r, \theta_{0}\right)} F(r, \theta)=\frac{\dot{D}(r, \theta)}{\dot{D}\left(r, \theta_{0}\right)}$.

One of the main advantages of this new approach is that now, a well-behaved fitting function can be found for $H_{\mathrm{L}}(r, \theta)$ (see below). But, in addition, a simple relationship with the 1D formalism is found:

$\phi_{\mathrm{an}}(r)=\frac{1}{2} \int_{0}^{\pi} \mathrm{d} \theta \sin \theta H_{\mathrm{L}}(r, \theta)$.

\section{Monte Carlo calculations}

To illustrate the ability of the modified formalism, the dosimetric characterization of the ${ }^{125}$ I selectSeed brachytherapy source, manufactured by Isotron Isotopentechnik (Berlin, Germany) and traded by Nucletron, has been carried out. 
We used the same geometry as in the paper of Karaiskos et al. [4]. It is a cylindrical source with the radioactive material embedded in a shell of a silver halide of thickness $3 \mu \mathrm{m}$ which coats a central silver rod. All these materials are encapsulated in a titanium tube with rounded edges. The active length of the source is $3.4 \mathrm{~mm}$.

We have performed an MC simulation of this seed situated in a water $\left(\rho=1 \mathrm{~g} / \mathrm{cm}^{3}\right)$ phantom of $100 \times 100 \times 100 \mathrm{~cm}^{3}$, using the PENELOPE code (v. 2006) [3].

The materials employed in the simulations were pure titanium $\left(\rho=4.54 \mathrm{~g} / \mathrm{cm}^{3}\right)$, pure silver $\left(\rho=10.50 \mathrm{~g} / \mathrm{cm}^{3}\right)$, dry air at sea level $\left(\rho=1.020479 \mathrm{mg} / \mathrm{cm}^{3}\right)$ and a silver halide with an atomic composition of $50 \% \mathrm{Ag}, 25 \% \mathrm{Br}$ and $25 \% \mathrm{Cl}(\rho=6.0 \mathrm{~g} /$ $\mathrm{cm}^{3}$ ). Unless explicitly indicated, all the simulations were performed using the following set of parameters: $C_{1}=C_{2}=0.05, W_{\mathrm{cc}}=10 \mathrm{keV}$ and $W_{\mathrm{cr}}=1 \mathrm{keV}$. The absorption energies for photons were fixed to $1 \mathrm{keV}$ and for electrons to $10 \mathrm{keV}$, in all materials. Finally, the maximum step size, $s_{\max }$, was fixed to one tenth of the thickness of each body in the geometry, as indicated in the code user manual [3].

Photons were generated in the active volume of the source and emitted in a random direction with an initial energy that was sampled according to the ${ }^{125}$ I photon energy spectrum quoted in [2].

The various factors and functions appearing in both 1D and $2 \mathrm{D}$ formalisms were calculated from the results of the MC simulations. Kerma and dose were obtained by scoring the energy deposited in voxels of various types.

To evaluate the air kerma strength, $S_{k}$, it was necessary to determine the dose in a plane perpendicular to the seed axis at the seed center. To do that, the seed was situated at the center of a cube of $500 \mathrm{~cm}$ of side, full of dry air, and the dose deposited along the seed transverse axis was calculated for $10^{11}$ initial photons. In this case, a cylindrical-planar grid with cylindrical shell voxels of widths $\Delta R=1 \mathrm{~cm}$ and $\Delta z=0.5 \mathrm{~cm}$ was used. In this case, the tracking parameters were fixed to $C_{1}=C_{2}=0.05, W_{c c}=50 \mathrm{keV}$ and $W_{c r}=5 \mathrm{keV}$, with absorption energies of $5 \mathrm{keV}$, for photons, and $50 \mathrm{keV}$, for electrons. According to TG-43 recommendation [2], X-rays generated at the titanium body were not considered.

To calculate the radial functions $g_{\mathrm{P}}(r)$ and $g_{\mathrm{L}}(r), M C$ simulations of the energy deposited in the water phantom, in cylindrical shell voxels with $\Delta R=0.04 \mathrm{~cm}$ and $\Delta z=0.02 \mathrm{~cm}$, for $10^{9}$ initial particles, were performed.

To evaluate the function $F(r, \theta)$, the energy was collected is spherical sector voxels with widths $\Delta r=0.05 \mathrm{~cm}$ and $\Delta \theta=0.4^{\circ}$. Simulations for $10^{9}$ initial photons were carried out.

Finally, for the function $\phi_{\mathrm{an}}(r)$, instead of the numerical integration proposed by TG-43 $[1,2]$, an MC point of view was adopted and we evaluated directly the spherical average dose rate

$\dot{D}_{\text {ave }}^{(2 \mathrm{D})}(r)=\frac{1}{2} \int_{0}^{\pi} \mathrm{d} \theta \sin \theta \dot{D}^{(2 \mathrm{D})}(r, \theta)$

and obtain the anisotropy factor as

$\phi_{\text {an }}(r)=\frac{\dot{D}_{\text {ave }}^{(2 \mathrm{D})}(r)}{\dot{D}^{(2 \mathrm{D})}\left(r, \theta_{0}\right)}$.

The advantage is that the spherical average $\dot{D}_{\text {ave }}^{(2 \mathrm{D})}(r)$ can be calculated, with a fair statistics, by collecting the energy absorbed in spherical shells, centered at the source center, and with a given $\Delta r$. In our case a value $\Delta r=0.04 \mathrm{~cm}$ was fixed. Figure 1 represents these spherical shells nearby the source. It must be noted that the energy is accumulated only in the part of the voxels not intersecting the seed volume. The volumes considered to evaluate the corresponding doses are

$V_{n}= \begin{cases}\frac{4 \pi}{3}\left[\left(r_{n}\right)_{\max }^{2}-R_{\mathrm{c}}^{2}\right]^{3 / 2}, & n=1, \\ \frac{4 \pi}{3}\left\{\left[\left(r_{n}\right)_{\max }^{2}-R_{\mathrm{c}}^{2}\right]^{3 / 2}-\left[\left(r_{n}\right)_{\max }^{2}-R_{\mathrm{c}}^{2}\right]^{3 / 2}\right\}, & n=2,3,4, \\ \frac{4 \pi}{3}\left[\frac{3}{4}\left(L^{2} R_{\mathrm{c}}+L R_{\mathrm{c}}^{2}+\frac{L^{3}}{2}\right)-\left(\frac{L^{2}}{4}-R_{\mathrm{c}}^{2}\right)^{3 / 2}\right], & n=5, \\ \frac{4 \pi}{3}\left[\left(r_{n}\right)_{\max }^{3}-\left(r_{n}\right)_{\max }^{3}\right], & n>5,\end{cases}$

where $R_{\mathrm{c}}$ is the radius of the capsule of the source $(0.4 \mathrm{~mm}$ in our case), $L$ refers to the length of the cylindrical part of the capsule $\left(3.7 \mathrm{~mm}\right.$ in our case) and $\left(r_{n}\right)_{\min }$ and $\left(r_{n}\right)_{\max }$ indicate the smaller and larger radii of the $n$-th spherical shell. It must be noted that $\left(r_{n}\right)_{\max }-\left(r_{n}\right)_{\min }=R_{\mathrm{c}}$.

\section{Fitting functions}

Different functions were fitted to the $M C$ results obtained for the quantities of interest. All these fits were done using the nonlinear least-squares Levenberg-Marquardt algorithm [11].

\section{Radial dose function}

Planning systems work with simple functions that fit the data calculated for $g_{\mathrm{P}}(r)$ and $g_{\mathrm{L}}(r)$. As indicated in TG-43 [1,2], polynomials up to the fifth degree in $r$ are commonly used. However, the range of validity of these polynomial approximations must to be taken into account explicitly, because their behavior, outside the fitting range, is completely

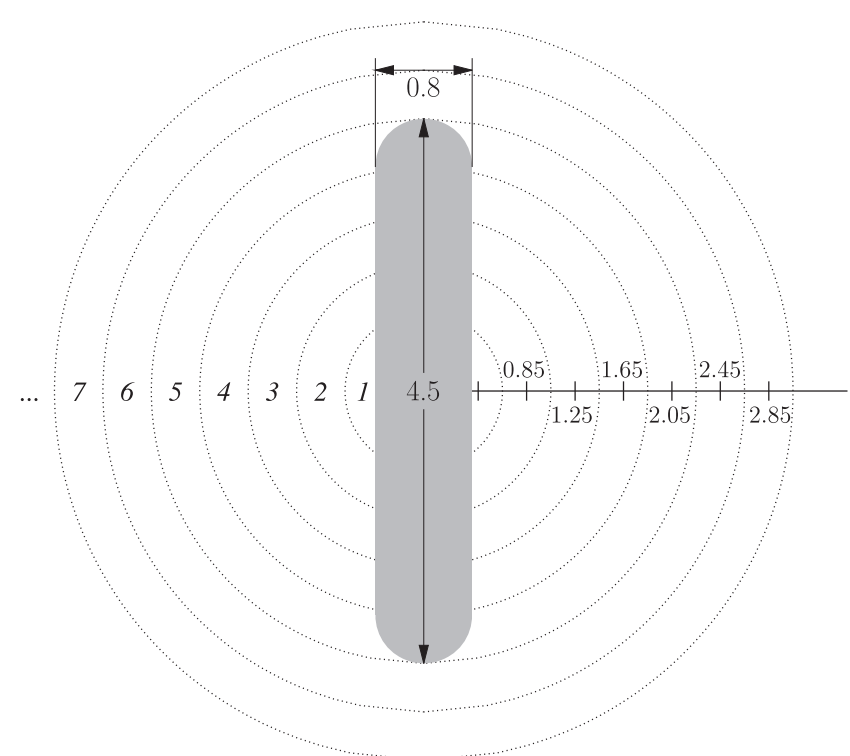

Figure 1 Scheme of the spherical shell voxels, nearby the source, used to determine the anisotropy factor $\phi_{\mathrm{an}}(r)$. Dimensions are in $\mathrm{mm}$. 
different than the asymptotic behavior of $g(r)$, which decreases almost exponentially. For that reason, other approximations were taken into account. As an example, we mention here the approach of Furhang and Anderson [10], who considered a combination of exponentials

$g^{(\text {Furh })}(r)=a_{0} \exp \left(-\mu_{1} r\right)\left[1+b_{0} \exp \left(-\mu_{2} r\right)\right]$,

in order to describe both the initial buildup region and the exponential decrease at large distances.

An improved data description can be obtained by using a fitting function that generalizes that of Furhang and Anderson [10]. According to the results obtained by Sabariego et al. [12], who developed a statistical model that predicts the buildup of successive generations of scattered photons for photon point sources, we considered the function:

$g^{(n m)}(r)=p_{n}(r) \exp \left(-\mu_{1} r\right)\left[1+q_{m}(r) \exp \left(-\mu_{2} r\right)\right]$,

where $p_{n}(r)$ and $q_{m}(r)$ are polynomials of degrees $n$ and $m$, with coefficients $\$ a \_k \$$ and $\$ b \_k \$$, respectively. Note that $g^{(\text {Furh })}(r) \equiv g^{(00)}(r)$. The idea is that the additional terms in the two polynomials take into account the contribution to both the buildup and the tail of the scattered photons.

We have considered for comparison also other approaches in the literature proposed by Moss [13], Meigooni et al. [14] and Taylor and Rogers [9]:

$g^{(\text {Moss })}(r)=a_{0} \frac{1+a_{1} \exp \left(-\mu_{1} r\right)}{1+a_{1} \exp \left(-\mu_{1} r\right)+b_{1} \exp \left(-\mu_{2} r\right)}$,

$g^{(\text {Meig) }}(r)=p_{5}(r) \exp \left(-\mu_{1} r\right)$

$g^{(\text {Tayl })}(r)=\frac{1}{r^{2}} p_{5}(r) \exp \left(-\mu_{1} r\right)$

\section{Anisotropy function}

Functional fits for $F(r, \theta)$ are not as popular as those for $g(r)$ and a common practice is to evaluate it using interpolation and extrapolation of tabulated values at certain points.

Polynomial fits have some inconveniences. For example, the fitting coefficients are largely correlated between themselves and usually have huge uncertainties. This makes that the estimations of $F(r, \theta)$ can be rather poor. Furhang and Anderson [10] proposed the function

$F^{\text {(Furh) }}(r, \theta)=1-\exp (c r)(a+b \theta) \cos \theta$.

Here we dealt with the new anisotropy function, $H_{\mathrm{L}}(r, \theta)$, defined in equation (5). For fixed $r$, this function can be expanded using the complete orthonormal set of Legendre polynomials of $\cos \theta$ as

$H_{\mathrm{L}}(r, \theta)=\sum_{l=0}^{\infty} \phi_{l}(r) P_{l}(\cos \theta)$,

where the expansion coefficients are given by

$\phi_{l}(r)=\frac{2 l+1}{2} \int_{0}^{\pi} \mathrm{d} \theta H_{\mathrm{L}}(r, \theta) P_{l}(\cos \theta)$.

Due to the angular symmetry properties of both $G_{\mathrm{L}}(r, \theta)$ and $F(r, \theta)$, only even $/$ terms do contribute to equation (16).
When $r$ becomes large, $F(r, \theta)$ tends to unity, whilst $G_{\mathrm{L}}(r, \theta)$ behaves as $r^{-2}$, for all $\theta$ values. Then, $H_{\mathrm{L}}(r, \theta)$ tends also to unity for large $r$ values. This is achieved if, for large $r, \phi_{2 m}(r)$ goes to zero, for $m \neq 0$, and $\phi_{0}(r)$ tends to unity. Moreover, if $\theta=\theta_{0}=\pi / 2, F(r, \theta)=1$ and then

$\sum_{m=0}^{\infty} \phi_{2 m}(r) P_{2 m}(0)=1$

for all $r$ values. In practice we used the expansion given by equation (16), truncated to a given maximum $M$,

$H_{\mathrm{L}}^{(M)}(r, \theta)=\sum_{m=0}^{M} \phi_{2 m}(r) P_{2 m}(\cos \theta)$,

to fit the $M C$ results for different $r$ values. To satisfy the sum rule of equation (18) we fixed the last term as

$\phi_{2 M}(r)=\frac{1}{P_{2 M}(0)}\left[1-\sum_{m=0}^{M-1} \phi_{2 m}(r) P_{2 m}(0)\right]$.

The results obtained with this function were compared to $H_{\mathrm{L}}^{(\text {Furh })}(r, \theta)=\frac{G_{\mathrm{L}}(r, \theta)}{G_{\mathrm{L}}\left(r, \theta_{0}\right)} F^{\text {(Furh) }}(r, \theta)$,

with $F^{(\text {Furh })}(r, \theta)$ given by equation (15).
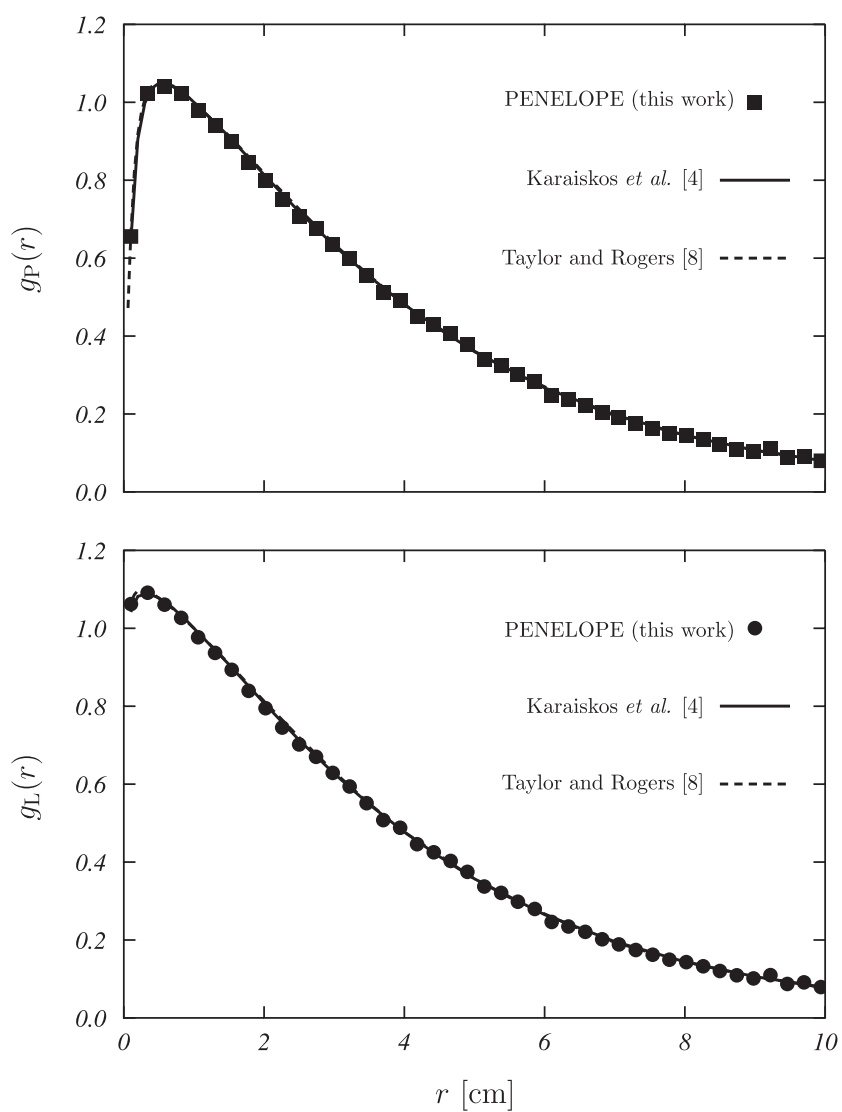

Figure 2 Radial dose functions $g_{\mathrm{P}}(r)$ (upper panel) and $g_{\mathrm{L}}(r)$ (lower panel) as a function of the distance to the source $r$ obtained in this work. The results of Karaiskos et al. [4] and Taylor and Rogers [8] are shown for comparison with solid and dashed lines, respectively. At the scale of the figure these two calculations almost overlap. Uncertainties are given with a coverage factor $k=1$ and are smaller than the symbols used. 


\section{Anisotropy factor}

Fits of the anisotropy factor are not usual, mainly because, as said above, the number of available data points is very small. Here we propose a 5-parameter fitting function defined as

$\phi_{\mathrm{an}}^{\mathrm{fit}}(r)=\frac{c}{b^{2}+(r-a)^{2}}+[1-d \exp (-f r)]$.

The first term is a Lorentzian function that permits to describe the behavior of $\phi_{\text {an }}$ at short distances to the source, while the second one provides the right behavior at long distances. This expression avoids the problem discussed above related to the polynomial approximations, which could present a wrong behavior out of the fitting range.

The new anisotropy function, $H_{\mathrm{L}}(r, \theta)$, allowed us to consider a second way to fit the anisotropy factor. In fact, substituting expansion (16) in equation (6) and using the orthogonality of the Legendre polynomials, we have

$$
\begin{aligned}
\phi_{\mathrm{an}}(r) & =\frac{1}{2} \int_{0}^{\pi} \mathrm{d} \theta \sin \theta H_{\mathrm{L}}(r, \theta) \\
& =\frac{1}{2} \sum_{m=0}^{\infty} \phi_{2 m}(r) \int_{0}^{\pi} \mathrm{d} \theta \sin \theta P_{2 m}(\cos \theta) \\
& =\frac{1}{2} \sum_{m=0}^{\infty} \phi_{2 m}(r) \delta_{m}=\phi_{0}(r) .
\end{aligned}
$$

The anisotropy factor coincides with the first term in the expansion (16). Therefore, an interesting feature of this expansion for the evaluation of the anisotropy function is that, when values of $\phi_{2 m}(r)$ are not well known (e.g. near the source), an adequate approximation which avoids possible interpolation/extrapolation errors is to use the values of $\phi_{\mathrm{an}}(r)$, well described by equation (22), instead of $\phi_{0}(r)$. This approach guarantees that the angular averaged dose rate remains accurate.

\section{Results and discussion}

\section{Air kerma strength and dose rate constant}

To calculate the air kerma strength, we performed a linear fit of the values obtained for the product $r^{2} \dot{K}(r)$ up to $r=100 \mathrm{~cm}$. We found $S_{K}=0.0218(1) \mathrm{U} \mathrm{Mbq}^{-1}$, in very good agreement (a relative difference of $0.6 \%$ ) with that found by Karaiskos et al. [4], $S_{K}=0.0217(1) \cup \mathrm{Mbq}^{-1}$.

The dose rate constant was calculated as the ratio $\Lambda=\dot{D}\left(r_{0}, \theta_{0}\right) / S_{K}$ and a value $\Lambda=0.953(4) \mathrm{cGy} \mathrm{h}^{-1} \mathrm{U}^{-1}$ was found. This value agrees within the uncertainty $(k=1)$ with the MC results of Karaiskos et al. [4], 0.954(5) $c G y h^{-1}$ $\mathrm{U}^{-1}$, and Lymperopoulou et al. [6], 0.950(14) $c G y \mathrm{~h}^{-1} \mathrm{U}^{-1}$ ("point" approach), and the two experimental measurements available (Anagnostopoulos et al. [5], 0.938(65) cGy $\mathrm{h}^{-1} \mathrm{U}^{-1}$, and Papagiannis et al. [7], 0.987(77) $\mathrm{cGy} \mathrm{h} \mathrm{h}^{-1} \mathrm{U}^{-1}$ ). Differences of $1.0 \%, 3.9 \%$ and $3.0 \%$, respectively, were

Table 1 Parameters found in the fits of the functions described in equations $(10)-(14)$ to the $M C$ results obtained for $g_{P}(r)$ (first row of each parameter) and $g_{\mathrm{L}}(r)$ (second row of each parameter). Also the $\chi^{2}$ per degree of freedom is given in each case.

\begin{tabular}{|c|c|c|c|c|c|}
\hline & $g^{\text {(Furh) }}(r)$ & $g^{\text {(Moss) }}(r)$ & $g^{(\text {Meig) }}(r)$ & $g^{(\text {Tayl) }}(r)$ & $g^{(12)}(r)$ \\
\hline \multirow[t]{2}{*}{$a_{0}$} & $1.247(4)$ & $1.036(6)$ & $0.36(1)$ & $0.0017(1)$ & $1.24(1)$ \\
\hline & $1.392(2)$ & $1.159(8)$ & $1.045(4)$ & $0.0002(2)$ & $1.31(2)$ \\
\hline \multirow[t]{2}{*}{$a_{1}$} & & $28(6)$ & $4.41(9)$ & $-0.083(2)$ & $0.279(9)$ \\
\hline & & $6.4(6)$ & $1.20(4)$ & $-0.012(3)$ & $0.23(3)$ \\
\hline \multirow[t]{2}{*}{$a_{2}$} & & & $-4.2(2)$ & $1.32(1)$ & \\
\hline & & & $-0.20(3)$ & $1.17(1)$ & \\
\hline \multirow[t]{2}{*}{$a_{3}$} & & & $3.0(2)$ & $0.02(5)$ & \\
\hline & & & $0.25(3)$ & $0.30(6)$ & \\
\hline \multirow[t]{2}{*}{$a_{4}$} & & & $-0.71(5)$ & $-0.019(3)$ & \\
\hline & & & $-0.043(7)$ & $-0.026(7)$ & \\
\hline \multirow[t]{2}{*}{$a_{5}$} & & & $0.075(6)$ & $0.00106(8)$ & \\
\hline & & & $0.0045(8)$ & $0.0014(2)$ & \\
\hline \multirow[t]{2}{*}{$\mu_{1}\left[\mathrm{~cm}^{-1}\right]$} & $0.236(3)$ & $1.6(1)$ & $1.07(1)$ & $0.22(3)$ & $0.398(3)$ \\
\hline & $0.270(3)$ & $1.05(5)$ & $0.82(2)$ & $0.36(4)$ & $0.385(5)$ \\
\hline \multirow[t]{2}{*}{$b_{0}$} & $-0.83(2)$ & & & & $-1.00(1)$ \\
\hline & $-0.237(7)$ & & & & $-0.24(1)$ \\
\hline \multirow[t]{2}{*}{$b_{1}$} & & $0.25(1)$ & & & $2.78(8)$ \\
\hline & & $0.36(1)$ & & & $0.21(5)$ \\
\hline \multirow[t]{2}{*}{$b_{2}$} & & & & & $-5.8(4)$ \\
\hline & & & & & $-0.9(2)$ \\
\hline \multirow{2}{*}{$\mu_{2}\left[\mathrm{~cm}^{-1}\right]$} & $5.9(2)$ & $-0.401(7)$ & & & $5.3(2)$ \\
\hline & $1.36(9)$ & $-0.373(5)$ & & & $3.1(3)$ \\
\hline \# Param & 4 & 5 & 7 & 7 & 7 \\
\hline \multirow[t]{2}{*}{$\chi^{2} /$ dof } & 44.3 & 13.3 & 11.0 & 1.1 & 1.1 \\
\hline & 7.6 & 3.7 & 1.3 & 1.0 & 1.0 \\
\hline
\end{tabular}
Uncertainties are given with a coverage factor $k=1$ and are expressed with a number between parentheses, i.e., 1.247 (4) means $1.247 \pm 0.004$. 

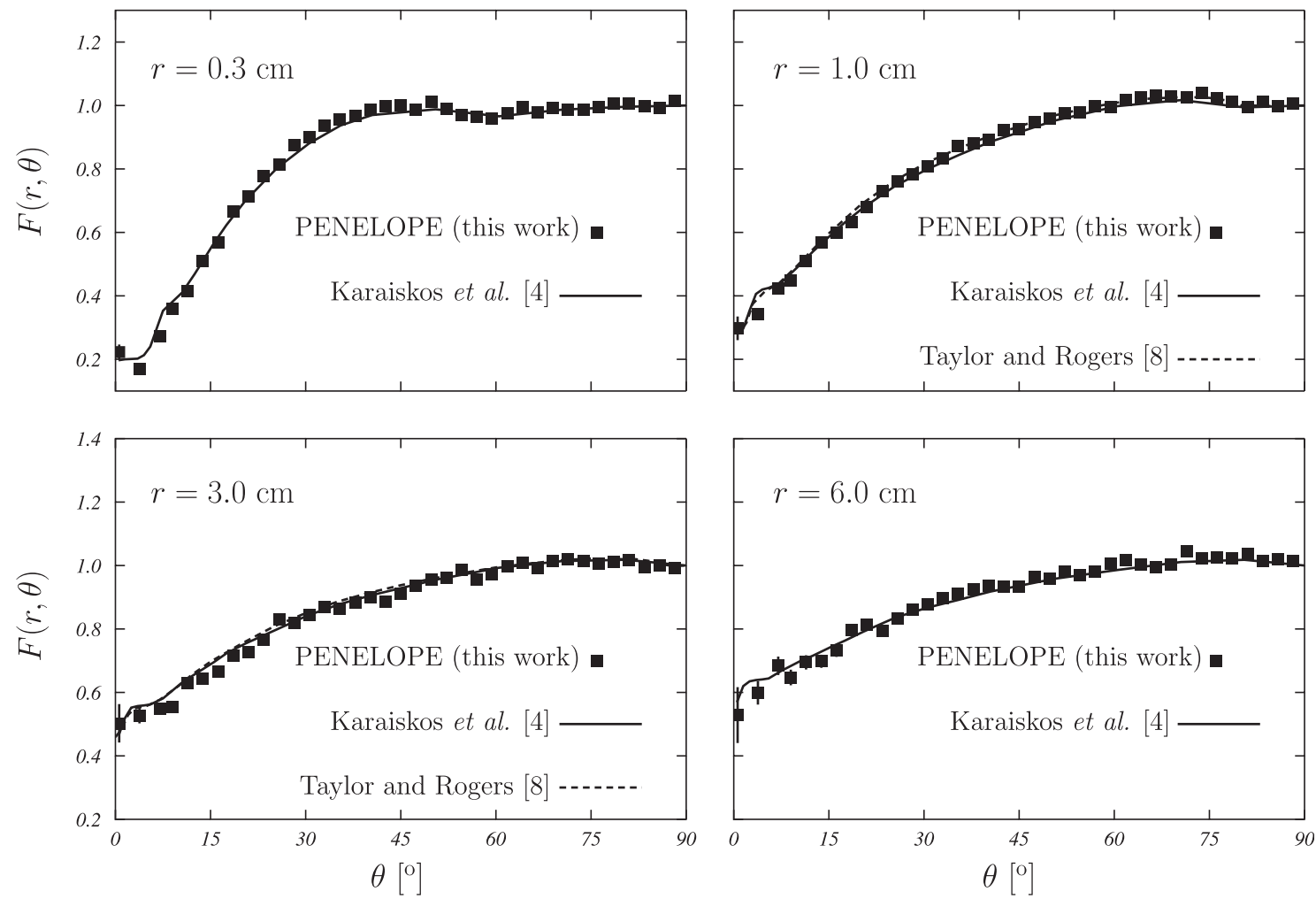

Figure 3 Comparison between the MC values of $F(r, \theta)$ (symbols), as a function of $\theta$, for $r=0.3,1,3$ and $6 \mathrm{~cm}$, and the results of Karaiskos et al. (2001) (solid curves). Uncertainties are given with a coverage factor $k=1$.

found with the MC calculations of Taylor and Rogers [8], 0.944(3) cGy h $\mathrm{h}^{-1} \mathrm{U}^{-1}$ ("point" approach) and 0.917(2) cGy $\mathrm{h}^{-1} \mathrm{U}^{-1}$ ("WAFAC" approach), and Lymperopoulou et al. [6], 0.925(14) cGy h ${ }^{-1} \mathrm{U}^{-1}$ ("WAFAC" approach).

\section{Radial dose function}

Results for $g_{\mathrm{L}}(r)$ and $g_{\mathrm{P}}(r)$ up to $r=10$ are illustrated in Fig. 2, where a comparison with those of Karaiskos et al. [4]
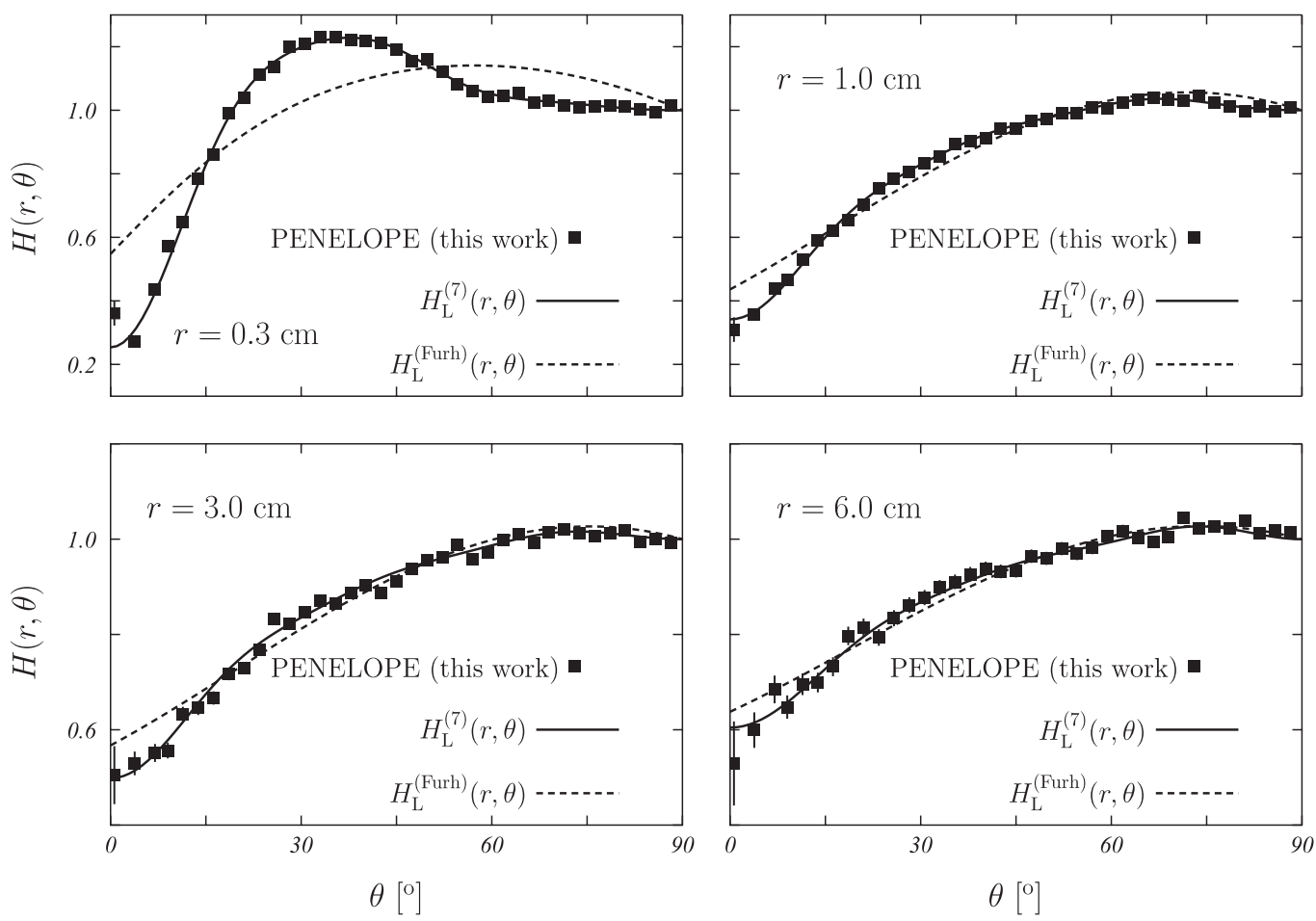

Figure 4 Comparison of the fits to the $M C$ results for $H_{\mathrm{L}}(r, \theta)$, using the $H_{\mathrm{L}}^{(\text {Furh })}(r)$ (dashed curves), given in equation (21), and $H_{\mathrm{L}}^{(7)}(r, \theta)$ (solid curves) for $r=0.3,1,3$ and $6 \mathrm{~cm}$. Uncertainties are given with a coverage factor $k=1$. 
Table 2 Coefficients of the fit to the MC values of the function $H_{\mathrm{L}}(r, \theta)$ using equation (19) with $M=7$, for some values of $r$. The values of the $\chi^{2}$ per degree of freedom for each fit are also given. Uncertainties are given with a coverage factor $k=1$ and are expressed with a number between parentheses, i.e., 1.0610 (7) means $1.0610 \pm 0.0007$.

\begin{tabular}{lllllllll}
\hline$r$ & $\phi_{0}$ & \multicolumn{1}{l}{$\phi_{2}$} & $\phi_{4}$ & \multicolumn{1}{l}{$\phi_{6}$} & $\phi_{8}$ & $\phi_{10}$ & $\phi_{12}$ \\
\hline 0.3 & $1.0610(7)$ & $0.033(1)$ & $-0.269(2)$ & $-0.280(2)$ & $-0.133(3)$ & $-0.056(3)$ & $-0.068(3)$ & 1.5 \\
0.5 & $0.9738(7)$ & $-0.188(1)$ & $-0.263(2)$ & $-0.102(2)$ & $-0.056(3)$ & $-0.063(3)$ & $-0.021(3)$ & 1.7 \\
0.7 & $0.9646(7)$ & $-0.226(1)$ & $-0.226(2)$ & $-0.060(2)$ & $-0.073(3)$ & $-0.036(3)$ & $-0.021(3)$ & 1.7 \\
1.0 & $0.9485(6)$ & $-0.238(1)$ & $-0.182(2)$ & $-0.052(2)$ & $-0.073(2)$ & $-0.021(3)$ & $-0.020(3)$ & 1.3 \\
1.5 & $0.9472(7)$ & $-0.232(1)$ & $-0.150(2)$ & $-0.043(2)$ & $-0.068(2)$ & $-0.013(3)$ & $-0.029(3)$ & 1.3 \\
2.0 & $0.9440(6)$ & $-0.223(1)$ & $-0.131(2)$ & $-0.042(2)$ & $-0.059(2)$ & $-0.011(3)$ & $-0.024(3)$ & 1.0 \\
3.0 & $0.9416(8)$ & $-0.204(2)$ & $-0.110(2)$ & $-0.039(3)$ & $-0.050(3)$ & $-0.011(3)$ & $-0.023(4)$ & 1.3 \\
4.0 & $0.9462(8)$ & $-0.189(2)$ & $-0.099(2)$ & $-0.034(3)$ & $-0.041(3)$ & $-0.014(3)$ & $-0.027(4)$ & 1.0 \\
6.0 & $0.958(1)$ & $-0.174(2)$ & $-0.092(3)$ & $-0.028(3)$ & $-0.045(4)$ & $0.004(4)$ & $-0.021(5)$ & 1.1 \\
\hline
\end{tabular}

(solid lines) and Taylor and Rogers [8] (dashed lines) is shown. The agreement between the three calculations is very good for both functions. Uncertainties quoted by Karaiskos et al. and by Taylor and Rogers are similar to ours. A table for the numerical values obtained in the present work for $g_{\mathrm{L}}(r)$ and $g_{\mathrm{P}}(r)$ is available online [15].

The $g^{\text {(Furh) }}(r)$ function proposed by Furhang and Anderson [10] and given by equation (10) was fitted to $M C$ results for $g_{\mathrm{P}}(r)$ and $g_{\mathrm{L}}(r)$. The values of the best fit parameters are given in Table 1. Two points deserve a comment. First, if the fits are performed by reproducing the tail, then the buildup regions is badly described, and vice versa. Second, the fits obtained by Furhang and Anderson [10] appeared to be much better than those found here. This was due to the fact that the data these authors fitted ranged between 0.5 and $5-7 \mathrm{~cm}$. If the fits carried out here are performed for $r$ values above $0.5 \mathrm{~cm}$, a nice description of the data is obtained but the buildup is completely missing.

Table 3 MC values of the anisotropy factor $\phi_{\text {an }}(r)$ obtained with PENELOPE. Uncertainties are given with a coverage factor $k=1$ and are expressed with a number between parentheses, i.e., 1.3714 (7) means $1.3714 \pm 0.0007$.

\begin{tabular}{llll}
\hline$r$ & $\phi_{\mathrm{an}}(r)$ & $r$ & \multicolumn{1}{c}{$\phi_{\mathrm{an}}(r)$} \\
\hline 0.0085 & $1.3714(7)$ & 0.7650 & $0.9518(9)$ \\
0.1250 & $1.4618(8)$ & 0.8050 & $0.9503(9)$ \\
0.1650 & $1.4109(8)$ & 0.8450 & $0.951(1)$ \\
0.2050 & $1.2657(7)$ & 0.8850 & $0.949(1)$ \\
0.2450 & $1.1364(7)$ & 0.9250 & $0.949(1)$ \\
0.2850 & $1.0741(7)$ & 0.9650 & $0.950(1)$ \\
0.3250 & $1.0353(7)$ & 1.005 & $0.949(1)$ \\
0.3650 & $1.0133(7)$ & 1.485 & $0.948(1)$ \\
0.4050 & $0.9961(7)$ & 2.005 & $0.945(2)$ \\
0.4450 & $0.9846(7)$ & 2.485 & $0.950(2)$ \\
0.4850 & $0.9756(8)$ & 3.005 & $0.947(2)$ \\
0.5250 & $0.9699(8)$ & 4.005 & $0.949(3)$ \\
0.5650 & $0.9653(8)$ & 5.005 & $0.955(4)$ \\
0.6050 & $0.9613(8)$ & 6.005 & $0.956(5)$ \\
0.6450 & $0.9582(8)$ & 8.005 & $0.951(7)$ \\
0.6850 & $0.9556(9)$ & 10.005 & $0.97(1)$ \\
0.7250 & $0.9544(9)$ & 16.085 & $0.99(4)$ \\
\hline
\end{tabular}

To improve the description of the MC data, the function $g^{(n m)}(r)$ proposed in equation (11), with $n=1$ and $m=2$, was fitted to the two $M C$ radial dose functions and the best fit parameters obtained are given also in Table 1 . The values of $\chi^{2}$ per degree of freedom around 1 indicate the accuracy of the fits provided by $g^{(12)}(r)$.

For completeness, also the functions $g^{(\text {Moss })}, g^{(\text {Meig) }}$ and $g^{(\text {Tayl) }}$, given by equations $(12)-(14)$, were fitted to our MC results. The values of the corresponding best fit parameters are shown in Table 1. The function $g^{\text {(Moss) }}$, proposed in [13], provides a reasonable description of $g_{L}$ (with a $\chi^{2} /$ dof of 3.7) but fails completely fitting $g_{p}$. This is due to the fact that $g^{\text {(Moss) }}$ shows its maximum at $r=0$. A similar situation occurs with $g^{\text {(Meig) }}$, proposed in [14], though in this case the description of $g_{L}$ is rather good (with a $\chi^{2} /$ dof of 1.3 ). Only the function $g^{(\text {Tayl) }}$, proposed in [9], and $g^{(12)}(r)$, proposed in the present work, are able to fit simultaneously the two $g$ functions and with the same accuracy (with a $\chi^{2} /$ dof $\sim 1$ ). The presence of two exponential functions makes $g^{(12)}(r)$ more flexible and this could be necessary for some other seeds.

\section{Anisotropy function}

Numerical results for $F(r, \theta)$ from our $M C$ simulations are available online [15]. In Fig. 3 some of these results are compared to those of Karaiskos et al. [4] (solid lines) and Taylor and Rogers [8] (dashed lines). In general, the agreement is reasonably good, with some deviation for small angles and short distances from the source. The uncertainties in our calculations are similar to those quoted by these authors.

As indicated above, instead of determining functional fits of $F(r, \theta)$, we performed fits of a new function $H_{\mathrm{L}}(r, \theta)$, defined in equation (5), using an expansion on Legendre polynomials given by equation (19). In Fig. 4 the results of these fits for $M=7$ and for $r=0.3,1,3$ and $6 \mathrm{~cm}$ (solid curves) are compared to the $M C$ values obtained in the simulations (symbols) and to the fits found using the function in equation (21), which is based on that proposed by Furhang and Anderson [10] to fit $F(r, \theta)$ (dashed curves). It is worth to point out that the fits obtained with $H_{\mathrm{L}}^{(M)}(r, \theta)$ are noticeably good, while those provide by $H_{\mathrm{L}}^{\text {(Furh) }}(r, \theta)$ fail to describe the MC data, mainly for small values of $r$ and/or $\theta$. 

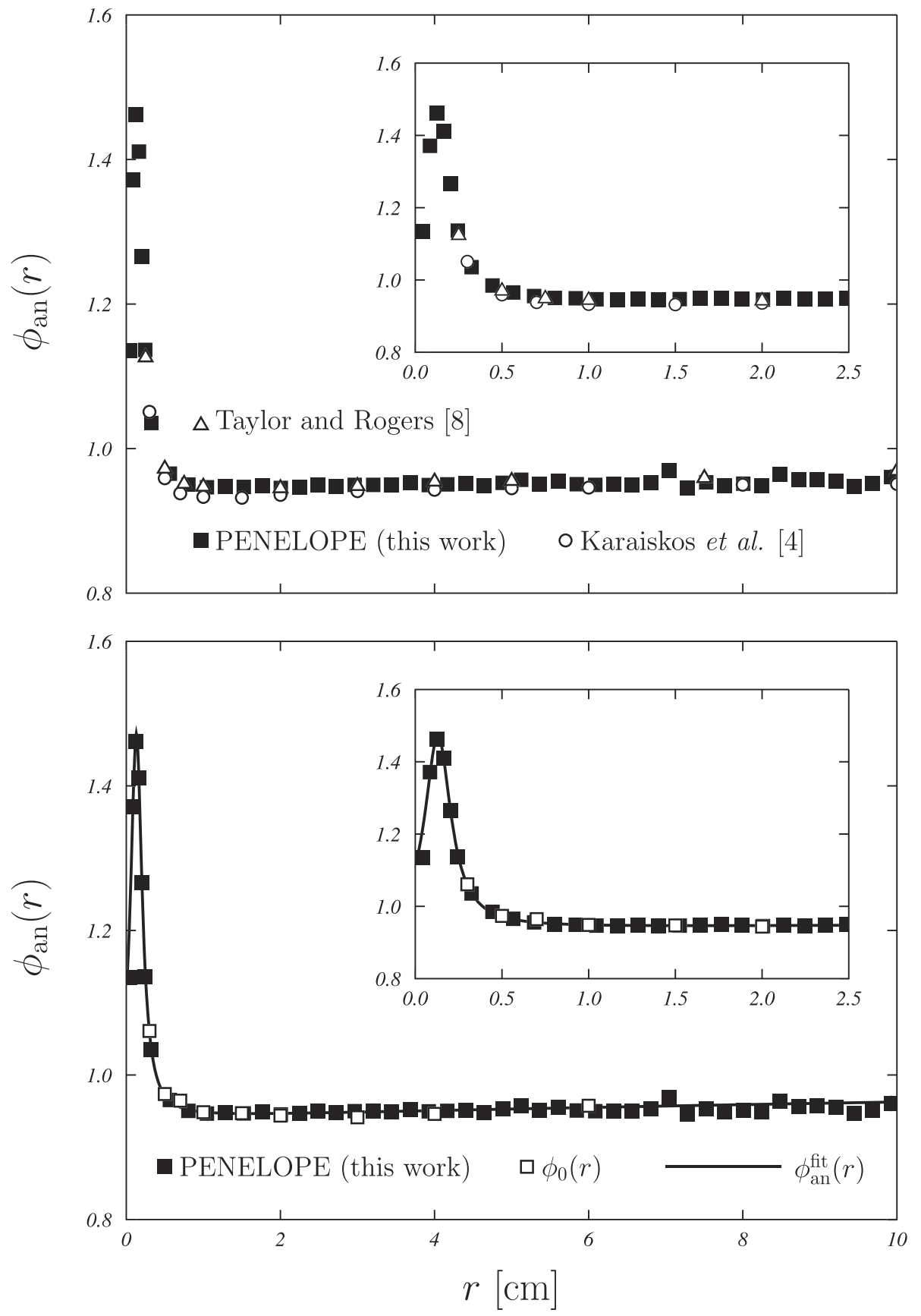

Figure $5 \quad M C$ values of the anisotropy radial factor (solid squares) compared to the results of Karaiskos et al. [4] (open circles) and Taylor and Rogers [8] (open triangles), in the upper panel, and to the fitted function given by equation (22) (solid line) and to the values of $\phi_{0}(r)$ (open squares), in the lower panel. Uncertainties are given with a coverage factor $k=1$ and are smaller than the symbols used.

The parameters $\phi_{2 m}(r)$, entering in equation (19), are given in Table 2, for different $r$ values ranging from 0.3 to $6 \mathrm{~cm}$. Also the values of $\chi^{2}$ per degree of freedom found in the corresponding fits are shown. These values point out the good quality of the fits found. In this respect it is important to note that, for each $r$ value, the fitting coefficients show very small correlations, contrary to what happens when one uses a polynomial function to carry out these fits.

\section{Anisotropy factor}

The $M C$ results obtained for the anisotropy factor, $\phi_{\mathrm{an}}$, are given in Table 3 for $r$ values up to $16 \mathrm{~cm}$, with particular detail nearby the source ( $r$ up to $2 \mathrm{~cm}$ ). In the upper panel of Fig. 5 we compare our results (solid squares) with those quoted by Karaiskos et al. [4] (open circles) and by Taylor and Rogers [8] (open triangles). Karaiskos et al. did not indicate either the way how they performed the calculation 
to obtain $\phi_{\mathrm{an}}(r)$ or the uncertainties of their calculation. Taylor and Rogers performed an integration of the dose rate for $\theta$ between 0 and $90^{\circ}$. The agreement is reasonably well, being a little bit better that of our results with those of Taylor and Rogers.

However, the lack of data closer to the source, that is for $r<0.25 \mathrm{~cm}$, do not allow the comparison in the region where the anisotropy factor changes significantly. This behavior of $\phi_{\mathrm{an}}(r)$ nearby the source was also observed by Rivard [16] in the ${ }^{125}$ I seeds models 6711 and 9011. This peak is due to the fact that, for small $r$, the spherical scoring voxels include points very close to the source, where the attenuation due to the water medium is strongly reduced. This makes $\phi_{\mathrm{an}}(r)$ to increase.

In the lower panel of the same figure, we compare the MC results with the fit obtained according to equation (22) which is plotted with a solid curve. The values of fitting parameters were: $a=0.1303(3), b=0.0912(5), c=0.00442(5)$, $d=0.0599(6)$ and $f=0.048(5)$ with a $\chi^{2}$ per degree of freedom of 5.0. We can see that the description of the data provided by this fitting function is very good.

Open squares in the lower panel of Fig. 5 represent the results obtained for $\phi_{0}(r)$ that are shown in Table 2. As indicated above, this values permit to describe the anisotropy factor in a direct way and, as we can see, the results confirm this point.

Some treatment planning systems do not permit the separated entry of both $g(r)$ and $\phi_{\text {an }}(r)$. In such case, the TG-43 protocol [2] proposes to use directly the product

$g_{\mathrm{X}}^{\prime}(r)=g_{\mathrm{X}}(r) \phi_{\mathrm{an}}(r)$

where $X$ stands for $L$ or $P$ approaches. The analytical fits performed in this work for the functions $g(r)$ and $\phi_{\text {an }}(r)$ (see equations (11) and (22)) allow a straightforward evaluation of this function at any distance.

\section{Conclusions}

In this work, a modification of the TG-43 protocol for seeds with cylindrical geometry has been proposed. The main point is the definition of a new anisotropy function and this permits a simpler description of the dependence of the dose rate with $r$ and $\theta$.

The ability of the modified protocol is analyzed for the Nucletron selectSeed brachytherapy source of ${ }^{125} \mathrm{I}$, for which the dosimetry is carried out using the MC code PENELOPE. Our results are in agreement with the available results.

The high statistics of our calculations allowed us to refine the values of some parameters previously reported, specially in the case of the anisotropy factor $\phi_{\mathrm{an}}(r)$ near the source.

New precise functional fits of the $M C$ results with simple expressions have been proposed. They provide an accurate description of the various functions, showing the right behavior outside the fitting range.

Usually, therapy planning systems for brachytherapy are based on data tables and constant parameters as inputs. For example, in the case of prostate brachytherapy treatments, the two parameters $S_{\mathrm{k}}$ and $\Lambda$ and the tables for $g(r)$, $F(r, \theta)$ and $\phi_{\text {an }}(r)$ are needed. The user can choose among 2D or 1D anisotropy calculations. In general, a strict table format is mandatory, being not possible to introduce a table with a larger spatial resolution. But recently, new planning systems allow to customize the input tables setup. The use of the results here quoted in these new planning systems may improve the dosimetric results significantly.

\section{Acknowledgments}

This work has been supported in part by the Junta de Andalucía (FQM0220) and by the Spanish DGI (FPA200914091-C02-02)

\section{References}

[1] Nath R, Anderson LL, Luxton G, Weaver KA, Williamson JF, Meigooni AS. Dosimetry of interstitial brachytherapy sources: recommendations of the AAPM radiation therapy Committee Task Group 43. Med Phys 1995;22:209-34.

[2] Rivard MJ, Coursey BM, DeWerd LA, Hanson WF, Huq MS, Ibbott GS, et al. Update of AAPM Task Group Nr. 43 Report: a revised protocol for brachytherapy dose calculations. Med Phys 2004;31:633-74.

[3] Salvat F, Fernández-Varea JM, Sempau J. PENELOPE - A code system for Monte Carlo simulation of electron and photon transport. Paris: NEA-OECD; 2006.

[4] Karaiskos P, Papagiannis P, Sakelliou L, Anagnostopoulos G, Baltas D. Monte Carlo dosimetry of the selectSeed ${ }^{125} \mathrm{I}$ interstitial brachytherapy seed. Med Phys 2001;28:1753-60.

[5] Anagnostopoulos G, Baltas D, Karaiskos P, Sandilos P. Thermoluminescent dosimetry of the selectSeed ${ }^{125} \mathrm{I}$ interstitial brachytherapy seed. Med Phys 2002;29:708-16.

[6] Lymperopoulou G, Papagiannis P, Sakelliou L, Karaiskos P, Sandilos P, Przykutta A, et al. Monte Carlo and thermoluminescence dosimetry of the new IsoSeed model 125.S17 125 interstitial brachytherapy seed. Med Phys 2005;32:3313-7.

[7] Papagiannis P, Sakelliou L, Anagnostopoulos G, Baltas D. On the dose rate constant of the selectSeed $125 \mathrm{I}$ interstitial brachytherapy seed. Med Phys 2006;33:1522-3.

[8] Taylor REP, Rogers DWO. An EGSnrc Monte Carlo-calculated database of TG-43 parameters. Med Phys 2008;35:4228-41.

[9] Taylor REP, Rogers DWO. More accurate fitting of ${ }^{125} \mathrm{I}$ and ${ }^{103} \mathrm{Pd}$ radial dose functions. Med Phys 2008;35:4242-50.

[10] Furhang EE, Anderson LL. Functional fitting of interstitial brachytherapy dosimetry data recommended by the AAPM Radiation Therapy Committee Task Group 43. Med Phys 1999; 26:153-60.

[11] Press WH, Teukolsky SA, Vetterling WT, Flannery BP. Numerical Recipes in Fortran. The Art of Scientific Computing. Cambridge: Cambridge University Press; 1992.

[12] Sabariego MP, Porras I, Lallena AM. Simple analytical expressions for the dose of point photon sources in homogeneous media. Phys Med Biol 2008;56:6113-28.

[13] Moss D. Technical note: improved analytical fit to the TG-43 radial dose function. Med Phys 2000;27:659-61.

[14] Meigooni AS, Zhang H, Perry C, Dini SA, Koona RA. Theoretical and experimental determination of dosimetric characteristics for brachyseed Pd-103, model Pd-1, source. Appl Radiat Isot 2003;58:533-41.

[15] http://fm197.ugr.es/selectSeed/.

[16] Rivard MJ. Monte Carlo radiation dose simulations and dosimetric comparison of the model 6711 and $9011^{125}$ I brachytherapy sources. Med Phys 2009;36:486-91. 